\title{
Serum Vitamin B12, Homocysteine and Methylmalonic Acid Levels in Patients With Parenchymal Neuro-Behçet's Syndrome
}

\author{
Berivan BİTIK,,${ }^{1}$ Abdurrahman TUFAN, ${ }^{2}$ Şehri ELBEG, ${ }^{2}$ Ridvan MERCAN,${ }^{2}$ Hamit KÜÇÜK, ${ }^{2}$ \\ Orhan KÜÇÜKŞAHIN, ${ }^{3}$ Mehmet Akif ÖZTÜRK, ${ }^{2}$ Seminur HAZNEDAROĞLU, ${ }^{2}$ Berna GÖKER ${ }^{2}$ \\ ${ }^{1}$ Department of Rheumatology, Ankara Training and Research Hospital, Ankara, Turkey \\ ${ }^{2}$ Department of Rheumatology, Medical Faculty of Gazi University, Ankara, Turkey \\ ${ }^{3}$ Department of Rheumatology, Medical Faculty of Yildirım Beyazıt University, Ankara, Turkey
}

\begin{abstract}
Objectives: This study aims to investigate the role of serum levels of vitamin B12 (VitB12), homocysteine (Hcy), and methylmalonic acid (MMA) in the development of parenchymal neuro-Behçet's syndrome (NBS) and to compare them with healthy controls and Behçet's syndrome (BS) patients without NBS.

Patients and methods: Serum VitB12, Hcy, and MMA were measured using enzyme-linked immunosorbent assay in 64 patients (36 males, 28 females; mean age 36 \pm 8.3 years; range 20 to 56 years) who met the criteria of the International Study Group for Behçet's Disease and 30 matched healthy controls (17 males, 13 females; mean age $35.76 \pm 9.6$ years; range 20 to 50 years). The study participants were grouped as BS patients with NBS, BS patients without NBS, and healthy controls.

Results: There was no significant difference between the groups regarding serum VitB12, Hcy, and MMA levels ( $p>0.05)$. Serum VitB12 levels were below the lower limit and serum Hcy levels were above the upper limit in all participants. MMA levels were normal in all participants. There was no correlation between the serum levels of VitB12, Hcy, and MMA and disease activity $(p>0.05)$

Conclusion: The results of this study suggest that there is no significant association between VitB12 deficiency and parenchymal NBS. Further studies are needed to investigate the underlying mechanisms of the variable effects of VitB12 deficiency on different clinical manifestations of BS. Keywords: Behçet's syndrome; homocystein; vitamin B12.
\end{abstract}

Neuro-Behçet's syndrome (NBS) is a rare manifestation of Behçet's syndrome (BS) with significant morbidity and mortality. NBS can be categorized as parenchymal ( $\mathrm{p}-\mathrm{NBS}$ ) or vascular involvement and it has been suggested that these two subtypes have different pathogenesis. ${ }^{1,2}$ There are no defined risk factors for $\mathrm{p}-\mathrm{NBS}$.

Vitamin B12 (VitB12) has a central role in the normal functioning of the nervous system. Vitamin B12 deficiency is associated with hematological, psychiatric, cognitive and neurological manifestations..$^{3-5}$ Therefore, early recognition of VitB12 deficiency is important to prevent progressive and irreversible neurological impairment. ${ }^{6}$ Vitamin B12 has two co-factor actions. The first is the conversion of propionylCoA to methylmalonyl-CoA and finally to succinylCoA. That pathway is suggested to be important for the nervous system. ${ }^{7}$ The second action is the conversion of homocysteine (Hcy) to form methionine. Serum concentrations of $\mathrm{Hcy}$ and methylmalonic acid (MMA) are elevated in VitB12 deficiency, due to a decreased rate of metabolism. Many patients with low VitB12 levels are not VitB12 deficient, so the measurement of the serum concentrations of Hcy and MMA appears to be more sensitive for the diagnosis of VitB12 
deficiency. 8,9 Therefore, in this study, we aimed to investigate the role of serum levels of VitB12, $\mathrm{Hcy}$, and MMA in the development of p-NBS and to compare them with healthy controls and BS patients without $\mathrm{p}$-NBS.

\section{PATIENTS AND METHODS}

The study was conducted at Gazi University School of Medicine between January 2010 and June 2014 and included 64 BS patients (36 males, 28 females; mean age $36 \pm 8.3$ years; range 20 to 56 years), fulfilling the diagnostic criteria of the International Study Group for Behçet's Disease, ${ }^{10}$ and 30 matched healthy volunteers (17 males, 13 females; mean age $35.76 \pm 9.6$ years; range 20 to 50 years). The exclusion criteria were the taking of any vitamin supplements in the previous six months, chronic alcoholism, diabetes mellitus, hypertension, hyperlipidemia, any liver disease, renal failure or pregnancy. The study protocol was approved by Gazi University School of Medicine Ethics Committee. A written informed consent was obtained from each patient. The study was conducted in accordance with the principles of the Declaration of Helsinki.

Study participants were grouped as p-NBS patients, BS patients without $\mathrm{p}-\mathrm{NBS}$, and healthy controls. Parenchymal NBS was defined as meningoencephalitis with or without spinal lesions. BS patients without $\mathrm{p}$-NBS were then sub-grouped according to their type of involvement such as mucocutaneous, posterior uveitis, and vascular involvement group. Mucocutaneous involvement included patients with oral and genital ulcers, papulopustular lesions with or without arthritis. Vascular involvement included patients with deep venous thrombosis, cerebral venous sinus thrombosis, and pulmonary vascular thrombosis.
The blood samples were collected in ethylenediaminetetraacetate containing tubes after 12 hours of fasting; they were centrifuged immediately at $2000 \mathrm{x} \mathrm{g}$ for 10 minutes at room temperature to prevent Hcy formation and leakage from erythrocytes. Vitamin B12 was measured by the enzyme-linked immunosorbent assay method using commercially available kit from SunRed (cataloge number: 201-12-1545, Shangai, China). Serum Hcy and MMA levels were measured by enzyme-linked immunosorbent assay using commercially available kit from EASTBIOPHARM (cataloge number: CK-E91289). Normal blood ranges were accepted as 5-14 $\mu \mathrm{mol} / \mathrm{L}$ for Hcy, $3.1-15.3 \mathrm{ng} / \mathrm{mL}$ for MMA, and $187-911 \mathrm{pg} / \mathrm{mL}$ for VitB12.

\section{Statistical analysis}

SPSS for Windows version 15.0 (SPSS Inc., Chicago, IL, USA) software was used to analyze the data. Statistical analyses were undertaken using the Mann-Whitney U test and KolmogorovSmirnov test to compare the differences in laboratory values, and these data are presented as median and range. A value of $\mathrm{p}<0.05$ was accepted as statistically significant (confidence interval: 95\%).

\section{RESULTS}

The demographic data of the study population are shown in Table 1. Parenchymal NBS was determined in 24 patients $(20$ with meningoencephilitis, four with isolated transverse myelitis). A total of six patients with $\mathrm{p}$-NBS had spinal cord involvement on magnetic resonance imaging. Blood tests were applied during the active disease phase in 10 out of 24 patients with p-NBS. There were 23 patients with mucocutaneous involvement, 12 patients with posterior uveitis, and five patients with vascular

Table 1. Demographic data of study population

\begin{tabular}{|c|c|c|c|c|c|c|}
\hline & \multicolumn{2}{|c|}{ BS patients with $p-N B S(n=24)$} & \multicolumn{2}{|c|}{ BS patients without $p-N B S(n=40)$} & \multicolumn{2}{|c|}{ Healthy controls $(n=30$} \\
\hline & $\mathrm{n}$ & Mean \pm SD & $\mathrm{n}$ & Mean \pm SD & $\mathrm{n}$ & Mean $\pm S D$ \\
\hline Age (year) & & $33 \pm 6.2$ & & $36 \pm 8.3$ & & $35.76 \pm 9.6$ \\
\hline \multicolumn{7}{|l|}{ Sex } \\
\hline Male & 17 & & 24 & & 17 & \\
\hline Female & 7 & & 16 & & 13 & \\
\hline
\end{tabular}


Table 2. Serum vitamin B12, homocysteine, and methylmalonic acid levels of study participants

\begin{tabular}{|c|c|c|c|c|c|c|c|}
\hline \multirow[t]{2}{*}{ Serum concentration } & \multicolumn{2}{|c|}{ BS patients with $p$-NBS $(n=24)$} & \multicolumn{2}{|c|}{ BS patients without $p$-NBS $(n=40)$} & \multicolumn{2}{|c|}{ Healthy controls $(n=30)$} & \multirow[b]{2}{*}{$p$} \\
\hline & Median & Min-Max & Median & Min-Max & Median & Min-Max & \\
\hline VitB12 (pg/mL) & 133.5 & $67.7-1153$ & 118.9 & $67.7-1200$ & 171.3 & $69-1600$ & Ns \\
\hline Hcy $(\mu \mathrm{mol} / \mathrm{L})$ & 19.8 & $8-80$ & 21.2 & $8.2-80$ & 24 & $6.3-80$ & Ns \\
\hline MMA (ng/mL) & 11.1 & $3.2-48$ & 12.1 & $5.9-48$ & 11.4 & $4.7-48$ & Ns \\
\hline
\end{tabular}

involvement (two deep venous thrombosis, two cerebral venous sinus thrombosis, and one pulmonary artery thrombosis). Patients with p-NBS who also had posterior uveitis were not included in the posterior uveitis group.

Twenty-six patients with BS (all were in the mucocutaneous group) were using $1 \mathrm{mg} /$ day colchicine. All patients with p-NBS were using one of the immunosuppressants including cyclophosphamide and azathiopurine along with steroid therapy. Twenty-five patients (39\%) with BS were accepted as clinically active.

The median serum levels of VitB12, Hcy, and MMA in the participants are shown in Table 2. There was no significant difference between the groups regarding serum VitB12, Hcy, and MMA levels $(p>0.05)$. Serum VitB12 levels were below the lower limit and serum Hcy levels were above the upper limit in all participants. Methylmalonic acid levels were normal in all participants. There was no significant difference between the sub-groups of BS regarding serum VitB12, Hcy, and MMA levels ( $p>0.05$ ) (Table 3). There was no correlation between the serum levels of VitB12, Hcy, and MMA and disease activity ( $p>0.05)$. There was no statistically significant difference in the serum levels of VitB12, Hcy, and MMA between patients receiving or not receiving colchicine.

\section{DISCUSSION}

Parenchymal NBS is one of the most devastating manifestations in BS and there is no well-defined risk factor for $\mathrm{p}$-NBS. To our knowledge, this is the first study to report the levels of serum VitB12, Hcy, MMA in patients with p-NBS. It is well known that VitB12 is an essential vitamin for the proper functioning and development of the nervous system. Vitamin B12 deficiency has been reported to result in several neurological manifestations. ${ }^{3,4,11}$

In this study, no significant difference was found between Behçet's patients with or without $\mathrm{p}-\mathrm{NBS}$ and healthy controls in respect of serum VitB12, Hcy, and MMA levels. Serum VitB12 levels were below the lower limit in all participants. However, this cannot be interpreted as VitB12 deficiency in the participants, because Hcy levels were high, while MMA levels were within normal limits. Many patients with low VitB12 levels may not be VitB12 deficient. Elevation in the serum concentrations of both Hcy and MMA appears to be more sensitive for the diagnosis of VitB12 deficiency than serum VitB12 levels alone., Elevated MMA level is more specific for detecting VitB12 deficiency than an isolated elevation in Hcy level. ${ }^{12}$ Vitamin B12 or folic acid deficiency can both cause the Hcy level to rise. Isolated elevations in serum Hcy without an elevation in serum MMA is an important diagnostic marker

Table 3. Serum vitamin B12, homocysteine, and methylmalonic acid levels in subgroups of Behçet's syndrome

\begin{tabular}{|c|c|c|c|c|c|c|c|c|c|}
\hline \multirow[t]{2}{*}{$\begin{array}{l}\text { Serum } \\
\text { concentration }\end{array}$} & \multicolumn{2}{|c|}{$\begin{array}{l}\text { BS patients with pNBS } \\
(\mathrm{n}=24)\end{array}$} & \multicolumn{2}{|c|}{$\begin{array}{l}\text { Mucocutenous group } \\
(\mathrm{n}=23)\end{array}$} & \multicolumn{2}{|c|}{$\begin{array}{l}\text { Posterior uveitis group } \\
\qquad(\mathrm{n}=12)\end{array}$} & \multicolumn{2}{|c|}{$\begin{array}{l}\text { Vascular group } \\
\qquad(\mathrm{n}=5)\end{array}$} & \multirow[b]{2}{*}{$p$} \\
\hline & Median & Min-Max & Median & Min-Max & Median & Min-Max & Median & Min-Max & \\
\hline VitB12 (pg/mL) & 133.5 & $67.7-1153$ & 120.5 & $69.7-1600$ & 245.7 & 69.7-1915 & 114.69 & $67.7-204$ & Ns \\
\hline $\mathrm{Hcy}(\mu \mathrm{mol} / \mathrm{L})$ & 19.8 & $8-80$ & 20.2 & $12.3-80$ & 34 & $10.1-80$ & 20.7 & $8.2-35$ & Ns \\
\hline $\mathrm{MMA}(\mathrm{ng} / \mathrm{mL})$ & 10.3 & $3.2-48$ & 11.5 & $6.5-48$ & 21.6 & $6-48$ & 8.5 & 5.9-17.5 & Ns \\
\hline
\end{tabular}


for folic acid deficiency. ${ }^{13}$ However, in clinical practice, it may be problematic to determine the VitB12 deficiency with a single blood sampling, because it has been reported that the serum levels of VitB12, Hcy, and MMA may fluctuate with time and short-term fluctuations of VitB12, MMA, and Hcy levels may obscure the presence of VitB12 deficiency. ${ }^{14}$ In addition, Hcy and MMA may also be normal in patients with hematological or neurological abnormalities responsive to pharmacological doses of VitB12. ${ }^{14}$

In VitB12 deficiency, the dominant neurological pathology is myelopathy in the cervical and thoracic posterior columns. ${ }^{11}$ Magnetic resonance imaging of the spinal cord may show contrast enhancement involving the dorsal or lateral columns in VitB12 deficiency. ${ }^{15}$ In this study, only six BS patients with spinal cord involvement were included and the data were not sufficient to reveal the contribution of VitB12 deficiency to the pathogenesis or to the severity of spinal disease manifestation in p-NBS. Our limited experience is inclined to check for serum VitB12 levels particularly in patients with isolated spinal involvement of $\mathrm{p}$-NBS and to give the VitB12 therapy regardless of serum Hcy or MMA if the levels are low. Colchicine is known to interfere with VitB12 metabolism by reducing intrinsicfactor-B12 receptors. ${ }^{13}$ Although the number of patients was low, no difference was found in respect of serum levels of VitB12, Hcy, and MMA between patients receiving or not receiving colchicine.

In this study, serum Hcy levels were elevated in BS patients as well as in healthy controls. However, no correlation was determined between serum levels of Hcy and disease activity. Homocysteine was reported to be higher in patients with BS, particularly in patients with active disease status compared to healthy controls. ${ }^{16,17}$ Elevated Hcy has also been reported to be associated with thrombosis in vascular BS in several studies. ${ }^{18-21}$ Cerebral venous sinus thrombosis frequently co-occurs with peripheral venous vascular events such as deep venous thrombosis and pulmonary vascular disease; therefore, serum Hcy levels are expected to be high in vascular NBS. ${ }^{22}$ Homocysteine is known to be toxic for endothelial cells and has also been found to be associated with lower cognitive scores and neurological disorders. ${ }^{23,24}$
There are some potential weaknesses of this study. First, the number of patients was low. Second, hematological parameters were not recorded. However, it is known that both neurological and cognitive dysfunctions in VitB12 deficiency often develop in the absence of hematological changes. ${ }^{25}$ Finally, serum levels of folic acid were not checked. Therefore, it is difficult to interpret the isolated elevation of Hcy levels in all participants.

In conclusion, the results of this study suggest that there is no significant association between VitB12 deficiency and p-NBS. Unlike the previously reported association in venous thrombosis, no association was found between serum Hcy and parenchymal neurological involvement in patients with BS. Further studies are needed to investigate the underlying mechanisms of the variable effects of VitB12 deficiency on different clinical manifestations of BS, particulary in cases of spinal cord involvement.

\section{Declaration of conflicting interests}

The authors declared no conflicts of interest with respect to the authorship and/or publication of this article.

\section{Funding}

The authors received no financial support for the research and/or authorship of this article.

\section{REFERENCES}

1. Akman-Demir G, Serdaroglu P, Tasçi B. Clinical patterns of neurological involvement in Behçet's disease: evaluation of 200 patients. The NeuroBehçet Study Group. Brain 1999;122:2171-82.

2. Siva A, Kantarci OH, Saip S, Altintas A, Hamuryudan V, Islak $C$, et al. Behçet's disease: diagnostic and prognostic aspects of neurological involvement. J Neurol 2001;248:95-103.

3. Healton EB, Savage DG, Brust JC, Garrett TJ, Lindenbaum J. Neurologic aspects of cobalamin deficiency. Medicine (Baltimore) 1991;70:229-45.

4. Leishear K, Boudreau RM, Studenski SA, Ferrucci L, Rosano C, de Rekeneire N, et al. Relationship between vitamin B12 and sensory and motor peripheral nerve function in older adults. J Am Geriatr Soc 2012;60:1057-63.

5. van Overbeek EC, Staals J, van Oostenbrugge RJ. Vitamin B12 and progression of white matter lesions. A 2-year follow-up study in first-ever lacunar stroke patients. PLoS One 2013;8:78100. 
6. Martin DC, Francis J, Protetch J, Huff FJ. Time dependency of cognitive recovery with cobalamin replacement: report of a pilot study. J Am Geriatr Soc 1992;40:168-72.

7. Tefferi A, Pruthi RK. The biochemical basis of cobalamin deficiency. Mayo Clin Proc 1994;69:181-6.

8. Lindenbaum J, Savage DG, Stabler SP, Allen RH. Diagnosis of cobalamin deficiency: II. Relative sensitivities of serum cobalamin, methylmalonic acid, and total homocysteine concentrations. Am J Hematol 1990;34:99-107.

9. Matchar DB, McCrory DC, Millington DS, Feussner JR. Performance of the serum cobalamin assay for diagnosis of cobalamin deficiency. Am J Med Sci 1994;308:276-83.

10. Criteria for diagnosis of Behçet's disease. International Study Group for Behçet's Disease. Lancet 1990;335:1078-80.

11. Kumar N. Neurologic aspects of cobalamin (B12) deficiency. Handb Clin Neurol 2014;120:915-26.

12. Stabler SP. Screening the older population for cobalamin (vitamin B12) deficiency. J Am Geriatr Soc 1995;43:1290-7.

13. Snow CF. Laboratory diagnosis of vitamin B12 and folate deficiency: a guide for the primary care physician. Arch Intern Med 1999;159:1289-98.

14. Solomon LR. Cobalamin-responsive disorders in the ambulatory care setting: unreliability of cobalamin, methylmalonic acid, and homocysteine testing. Blood 2005; 105:978-85.

15. Locatelli ER, Laureno R, Ballard P, Mark AS. MRI in vitamin B12 deficiency myelopathy. Can J Neurol Sci 1999;26:60-3.

16. Taysi S, Sari RA, Dursun H, Yilmaz A, Keles M, Cayir $\mathrm{K}$, et al. Evaluation of nitric oxide synthase activity, nitric oxide, and homocysteine levels in patients with active Behcet's disease. Clin Rheumatol
2008;27:1529-34.

17. Ozkan Y, Yardim-Akaydin S, Sepici A, Engin B, Sepici V, Simşek B. Assessment of homocysteine, neopterin and nitric oxide levels in Behçet's disease. Clin Chem Lab Med 2007;45:73-7.

18. Aksu K, Turgan N, Oksel F, Keser G, Ozmen D, Kitapçioğlu G, et al. Hyperhomocysteinaemia in Behçet's disease. Rheumatology (Oxford) 2001;40:687-90.

19. La Regina M, Orlandini F, Prisco D, Dentali F. Homocysteine in vascular Behcet disease: a meta-analysis. Arterioscler Thromb Vasc Biol 2010;30:2067-74.

20. Ateş A, Aydintuğ O, Olmez U, Düzgün $N$, Duman M. Serum homocysteine level is higher in Behçet's disease with vascular involvement. Rheumatol Int 2005;25:42-4.

21. Feng L, Isaac V, Sim S, Ng TP, Krishnan KR, Chee MW. Associations between elevated homocysteine, cognitive impairment, and reduced white matter volume in healthy old adults. Am J Geriatr Psychiatry 2013;21:164-72.

22. Tascilar K, Melikoglu M, Ugurlu S, Sut N, Caglar E, Yazici H. Vascular involvement in Behçet's syndrome: a retrospective analysis of associations and the time course. Rheumatology (Oxford) 2014;53:2018-22.

23. Undas A, Brozek J, Szczeklik A. Homocysteine and thrombosis: from basic science to clinical evidence. Thromb Haemost 2005;94:907-15.

24. Ansari R, Mahta A, Mallack E, Luo JJ. Hyperhomocysteinemia and neurologic disorders: a review. J Clin Neurol 2014;10:281-8.

25. Lindenbaum J, Healton EB, Savage DG, Brust JC, Garrett TJ, Podell ER, et al. Neuropsychiatric disorders caused by cobalamin deficiency in the absence of anemia or macrocytosis. $\mathrm{N}$ Engl $\mathrm{J}$ Med $1988 ; 318: 1720-8$. 